\title{
Viele Leistungen können delegiert werden
}

\begin{abstract}
Die Allgemeinen Bestimmungen des EBM sehen unter Punkt I.2.2 vor, dass eine Nr. nur berechnet werden kann, wenn der Arzt die für die Abrechnung relevanten Inhalte „gemäß $\$ \$ 14 a, 15$ und $₫ 25$ Bundesmantelvertrag-Ärzte (BMV-Ä) persönlich erbringt“. Das klingt etwas kompliziert, zumal hier auf den BMV-Ä verwiesen wird, der nicht unbedingt zur Gewohnheitslektüre der Vertragsärzte zählen dürfte. Die Beachtung der Auflage ist aber unumgänglich - allein schon des-
\end{abstract} halb, weil man ansonsten unvermittelt in eine Plausibilitätsprüfung nach Zeitvorgaben verwickelt werden könnte. Bei den persönlich zu erbringenden Leistungen sieht der EBM nämlich solche Zeitvorgaben vor. Überschreitet man 12 Stunden an einzelnen Tagen oder 780 Stunden im Quartal, droht ein Regress oder sogar der Staatsanwalt.

\section{MMW-KOMMENTAR}

Die vertragsärztliche Tätigkeit muss persönlich ausgeübt werden - doch dies gilt auch als gegeben, wenn ärztliche Leistungen von

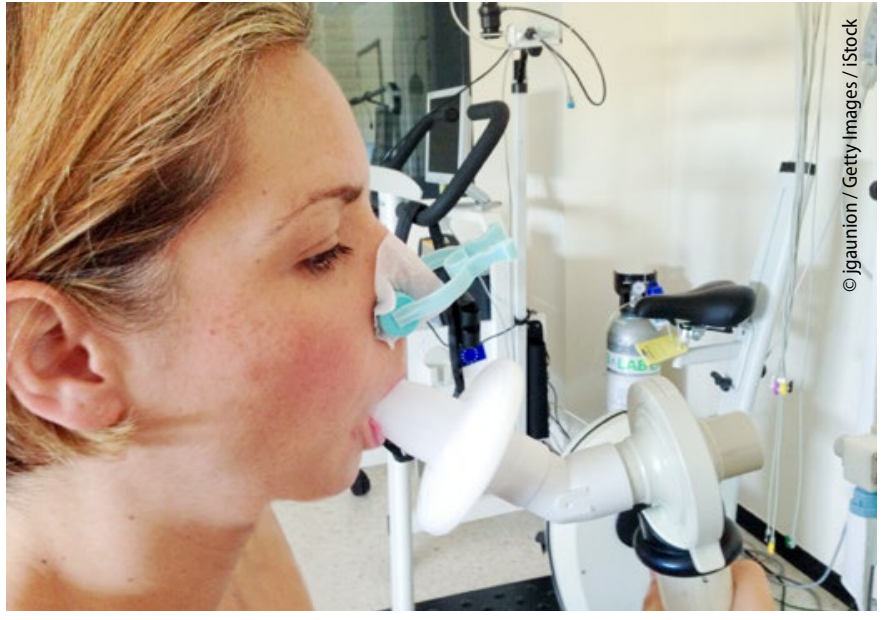
Eine Lufu kann an einen Mitarbeiter delegiert werden - sie schlägt
aber trotzdem mit drei Minuten im ärztlichen Zeitbudget zu Buche.

einbarung gemäß § 28 Abs. 1 $S G B V$ geregelt. Darin finden sich z. B. intramuskuläre und subkutane Injektionen und Impfungen, intravenöse Injektionen und das Anlegen einer Infusion, allgemeine Laborleistungen wie Blutzuckermessung, Urintests, kapilläre oder venöse Blutentnahmen, Wundversorgung und Verbandwechsel oder auch spirografische Untersuchungen. Im EBM wird die Möglichkeit der Delegation bei der Zuordnung der Plausibilitätszeiten berücksichtigt. Eine Lungenfunktionsprüfung z. B. schlägt

genehmigten Assistenten oder angestellten Ärzten erbracht werden, z. B. in einer Nebenbetriebsstätte oder in Abwesenheit des Vertragsarztes. Diese Leistungen werden dem Praxisinhaber zugerechnet, was aber ggf. erst nachträglich bei einer Plausibilitätsprüfung berücksichtigt werden kann.

Persönliche Leistungen sind auch Hilfeleistungen entsprechend qualifizierter nichtärztlicher Mitarbeiter, die der Vertragsarzt bzw. ein Assistent oder ein angestellter Arzt anordnet und fachlich überwacht. Eine Liste der auf diese Weise delegierbaren Leistungen haben die KBV und Kassen 2013 in einer Ver- mit drei Minuten zu Buche, ein Verband nach Nr. 02300 mit drei Minuten und einer nach Nr. 02301 mit sechs Minuten. Hier sieht man, dass diese Leistungen eben nicht vollständig delegierbar sind.

Eine Elektrotherapie nach Nr.02511 hingegen hat gar keine Zeitvorgabe und ist deshalb komplett an nicht-ärztliches Personal delegierbar. Das ist allerdings keine generelle Regel: Auch die unvorhergesehene Inanspruchnahme nach den Nrn.01 100 und 01101 oder der dringende Besuch im Heim nach Nr. 01415 haben keine Zeitvorgaben aber die muss der Arzt selbst erledigen.

\section{GOÄ: So rechnet man korrekt analog ab}

$\$ 6$ Abs. 2 der GOÄ sieht vor, dass ärztliche Leistungen, die sich nicht im Gebührenverzeichnis finden, entsprechend einer nach Art, Kosten- und Zeitaufwand gleichwertigen Leistung berechnet werden können. Mit den Jahrzehnten ist diese Regelung immer bedeutender geworden, da immer neue Leistungen in der Medizin auftauchen, die GOÄ aber einfach nicht reformiert wird. Nicht immer ist es einfach, die richtige Analogziffer zu finden - und private Krankenversicherungen und Beihilfestellen stellen sich immer öfter quer. Es ist deshalb wichtig, sorgfältig vorzugehen und ggf. Vorschläge der Bundesärztekammer oder von Gerichten einzubeziehen.

\section{MMW-KOMMENTAR}

Eine analoge Bewertung kann also allein aus der GOÄ abgeleitet werden. Der Arzt muss eine andere Leistung heraussuchen, die in etwa gleichwertig ist. Die Bundesärztekammer bietet ein Analogverzeichnis, das mit den Bundesministerien für Gesundheit und Inneres sowie dem PKV-Verband abgestimmt wird - es ist also quasi eine GÖ̈-Ergänzung, ohne dass es Rechtsverbindlichkeit hat. Ein Beispiel für eine solche Empfehlung ist die Nr. 659 A, die zur Abrechnung der mittlerweile zur Kassenleistung erklärten kontinuierlichen Blutzuckermessung über mindestens 18 Stunden herangezogen werden kann. Da hier - im Gegensatz zum EBM nicht zwischen den verschiedenen zur Verfügung stehenden Methoden differenziert wird, können bei der Abrechnung alle Systeme zugrunde gelegt werden - auch die im Moment im EBM noch ausdrücklich ausgeschlossenen Flash-Systeme.

Dr. Gerd W. Zimmermann, Hofheim 